\title{
DÜBLIN
}

Technological University Dublin ARROW@TU Dublin

\section{Advances in control of food mixing operations}

\author{
Patrick J. Cullen \\ Technological University Dublin, patrick.j.cullen@tudublin.ie \\ Serafim Bakalis \\ Technological University Dublin \\ Carl Sullivan \\ Technological University Dublin, carl.sullivan@tudublin.ie
}

Follow this and additional works at: https://arrow.tudublin.ie/schfsehart

Part of the Food Processing Commons, and the Medicine and Health Sciences Commons

\section{Recommended Citation}

Patrick Cullen, Serafim Bakalis, Carl Sullivan, Advances in control of food mixing operations, Current Opinion in Food Science, Volume 17, 2017, Pages 89-93, ISSN 2214-7993, DOI: 10.1016/ j.cofs.2017.11.002.

This Article is brought to you for free and open access by the School of Food Science and Environmental Health at ARROW@TU Dublin. It has been accepted for inclusion in Articles by an authorized administrator of ARROW@TU Dublin. For more information, please contact arrow.admin@tudublin.ie, aisling.coyne@tudublin.ie, gerard.connolly@tudublin.ie.

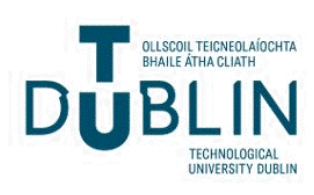




\section{Advances in control of food mixing operations Patrick Cullen $^{1,2}$, Serafim Bakalis ${ }^{1,2}$ and Carl Sullivan ${ }^{1}$}

\begin{abstract}
Mixing is a ubiquitous unit operation within the food industry and employed not only to combine multiple ingredients, but also to control reactions and modify the structure of foods. Failure of the mixing process may result in poor quality, organoleptic or functional attributes of foods along with product safety concerns. The industry is increasingly adopting automation and advanced control strategies such as Process Analytical Technology. Sensors can provide both insights into the complex mechanisms of mixing along with effective control. Here we discuss some recent advances in the control of food mixing operations.
\end{abstract}

\author{
Addresses \\ ${ }^{1}$ School of Food Science and Environmental Health, Dublin Institute of \\ Technology, Ireland \\ ${ }^{2}$ Department Chemical and Environmental Engineering, University of \\ Nottingham, UK \\ Corresponding author: Cullen, Patrick (pjcullen@dit.ie)
}

Current Opinion in Food Science 2017, 17:89-93

This review comes from a themed issue on Food engineering and processing

Edited by Colette Fagan

For a complete overview see the Issue

Available online 8th November 2017

http://dx.doi.org/10.1016/j.cofs.2017.11.002

2214-7993/@ 2017 Elsevier Ltd. All rights reserved.

\section{Introduction}

Food is the oldest and most widely employed application of mixing, with infinite combinations of mixed ingredients possible to meet consumer demands of nutrition, taste and texture [1]. Mixing is a ubiquitous unit operation carried out in the process and chemical industries to reduce inhomogeneities in product composition to an acceptable level and provide a more uniform processing environment or a more uniform product [2]. Such aims include reducing gradients of concentration, temperature or solids mass fractions. However for the food industry, mixing is also employed to modify the structure of foods. An important aspect for food processing is the development of mouthfeel or texture through mixing or conversely the potential negative influence of mixing on the sensory characteristics of products. Failure of the mixing process(s) may result not only in poor quality, organoleptic or functional attributes of foods but under-processed foods leading to food safety risks. Mixing is of paramount importance in assuring product safety through controlling the homogeneity of process treatments such as heating. For the food industry, the effectiveness of mixing should be ultimately assessed in the context of the quality and safety profiles of the end product.

\section{Defining mixing, scales of scrutiny and sampling}

As noted above, defining the mixing process will depend on the process objective(s). However looking at the spatial distribution of a species (ingredient, component, temperature etc), one could define a perfect mixture as one where each sample obtained contains exactly the same concentrations as the average for the whole batch, which is one of zero variance between samples [2]. However this is not a process reality given the probability of forming such a mixture by a stochastic process is extremely low. Therefore, we need to define both a measure of 'mixness' and an appropriate scale of scrutiny of sampling. Once the mixing objectives and values of adequate mixing have been established, the physical properties of the food will determine the type of mixer suitable to complete the task (Figure 1), with all these factors influencing or restricting the type of control strategy. Broadly speaking mixing is divided between fluid mixing and granular material blending, which display very different behaviours, require different mixing approaches and consequently associated control strategies.

\section{Single point, multipoint and imaging}

At production scales, the composition of the whole mixture cannot be determined at a single time point, so sampling is often used to assess the state of mixedness [2]. Given that mixing and blending processes are inherently concerned with the spatial distribution of the target species, capturing of data from multiple locations (stratified sampling), is important to obtain representative sampling. Similarly, when employing sensors to monitor the mixing process it is also beneficial to monitor at multiple locations. Multipoint and imaging sensors are emerging which are directly targeted at meeting the challenges of monitoring blend uniformity within mixers. Imaging techniques, particularly those which can identify chemical composition (chemical imaging) can be particularly useful to controlling the process and gaining mechanistic insights. Stream sampling, where the sample or measurement is taken when the mixture or blend is exiting the mixer can also offer a number of sampling and technical advantages for process monitoring and control. 
Figure 1

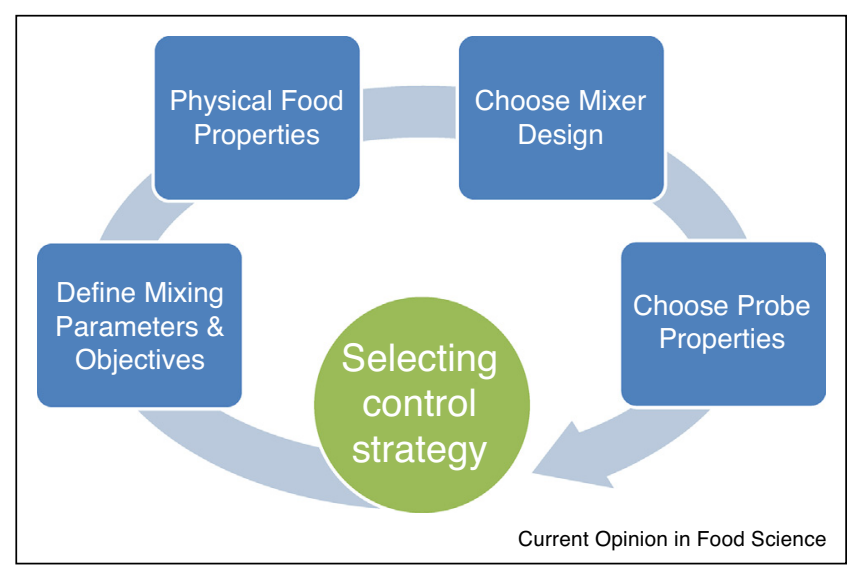

Influencing factors on selection of a control strategy for food mixing.

The objective of this opinion article is to present an overview of the status and trends in sensors to control mixing and blending processes. A select few examples of novel sensors which may be advantageous for defined food mixing processes are briefly discussed.

\section{Powders and granulate materials}

The food industry is increasing developing powders as nutritional products, such as powdered infant formula (PIF) and dietary supplements, where the composition of the blended ingredients is critical. The challenges of assuring blend homogeneity are similar to those experienced by the small molecule pharmaceutical industry.

\section{NIR spectroscopy}

Diffuse reflectance near infrared (NIR) spectroscopy is particularly suited to in-line monitoring offering noninvasive analytical optics. The technology is increasingly gaining acceptance and adoption for regulated processes for measuring product moisture content, ingredient identity and granule/particle homogeneity [3-6]. Indeed the European Medicines Agency (EMA) is working on producing guidelines on the technology as it is considered the most advanced and frequently encountered of the promising Process Analytical Technology (PAT) efforts aimed at controlling pharmaceutical manufacturing [7]. The principal drivers for its adoption as a blending monitoring technique is its non-invasive nature and the ability to operate under real-time condition combined with the fact that $\mathrm{C}-\mathrm{H}, \mathrm{N}-\mathrm{H}$ and $\mathrm{O}-\mathrm{H}$ chemical bonds absorb strongly in the NIR wavelength. In fact, current challenges are more focused on approaches integrate NIR systems to production lines to facilitate continuous process monitoring. Recent technological advances including; the development of microelectromechanical systems (MEMS) based in-line designs, wireless data acquisition, and real-time multi-point systems are particularly useful for the challenges of integrating NIR systems directly within static or rotating mixers or at the discharge point facilitating stream sampling. Progress in chemometrics, data-mining approaches, models and statistical process has improved the performance and robustness of the technology. Figure 2 shows a multi-point NIR spectrometer employing a four-pixel silicon-based detector, where each pixel works independently and records a light signal input from the four independent fibre optic connectors. The designs allows for full spectra to be recorded simultaneously from the four channels in the 1515-2100 nm spectral region with $5 \mathrm{~nm}$ wavelength increments. The efficacy of the approach for chemical identification and quantification of granule blends was recently demonstrated [8]. In a further study the technology was employed to determine the carbohydrate and protein content of PIF samples under both static and motion conditions, simulating industrial scenarios such as blending [9]. Strong predictions were obtained under both conditions, demonstrating the system's potential for in/on-line applications. By coupling light collimators to fibre-optics higher operating distances can be achieved offering greater flexibility in system integration within process vessels and monitoring through viewing windows.

\section{Raman spectroscopy (RS)}

Raman spectroscopy is based on monochromatic incident light generally from a laser source in the visible, NIR or near-UV range. RS fibre optic probes have been assessed to study blend uniformity of powders in planetary bowl mixers, bin blenders [10] and V-blenders [11] with the approach proving an effective predictor of mixing endpoint. As with NIR, the non-contact nature of RS probes means that they can be interfaced with the product via a sapphire window of process unit operations. Results to date point to RS as an effective and robust PAT technology, suitable for blend monitoring [12]. Future adoption of RS as a PAT tool for process monitoring including blending is likely as capital costs of the technology continue to fall and more reliable probes with real time in-line analytical capabilities are emerging. RS is expected to become a successful PAT tool for solid blending operations [7] and is suited to food processing conditions.

\section{Laser-induced breakdown spectroscopy (LIBS) and X- ray fluorescence (XRF) spectrometry}

Laser-induced breakdown spectroscopy (LIBS) and Xray fluorescence (XRF) spectrometry are emerging process analytical techniques for characterisation and identification of materials. One emerging challenges for quality assurance of food powders is the distribution of trace elements for example iron, zinc, copper and magnesium content of PIF. Techniques which could measure trace elements in the ppm range directly from the blending step would facilitate improved process control and quality assurance. As an entirely optical technique LIBS can be considered a PAT for qualitative and quantitative chemical analysis. LIBS is an optical emission spectroscopy 


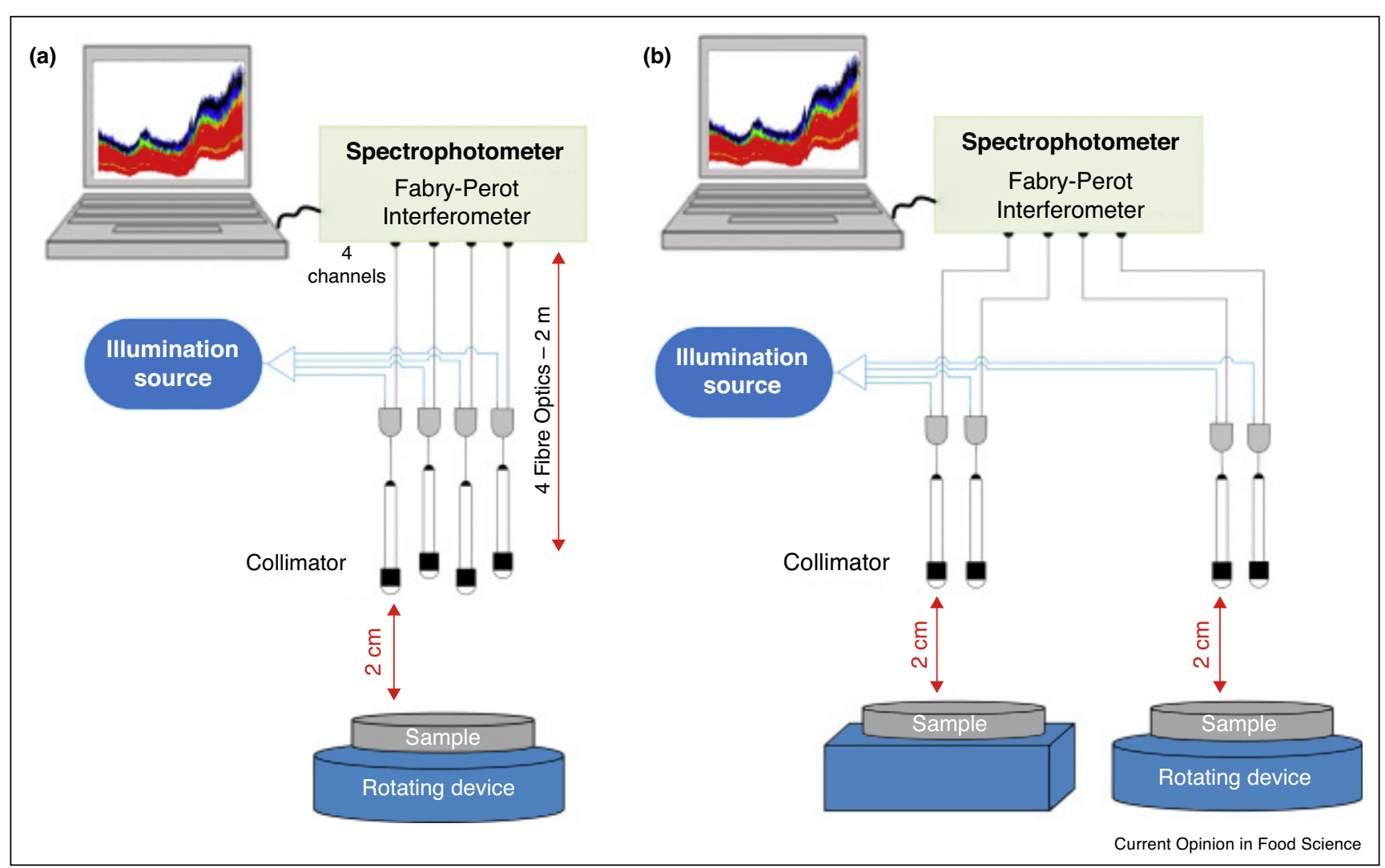

Multipoint spectrometer with four reflectance probes attached to an illumination source. Simultaneous recording of spectra from moving and stationary samples.

(OES) technique where a laser pulse is fired at a target and a small amount of material is ablated, atomised and ionised to produce a plasma plume. The resultant plasma, a mixture of atoms, ions and free electrons, lose energy and emit light at characteristic spectra for the target's elements. Attractive features include; multi-elemental analysis, fast response, remote sensing and little to no sample preparation. The technology is very new within food analysis, however, a diverse range of at-, on- and in-line applications are feasible mainly for mineral composition analysis, but also for a wide range of qualitative determinations such as authenticity control, sample discrimination, bacteria contamination, depth profiling and elemental surface mapping [13]. It is the potential to provide spatial analysis of material elements that would be useful for blend monitoring and control, particularly for trace elements. Potential applications include metal analysis of powdered infant formula. Coupling LIBS with motorised stages or presentation of the material as a flow/stream allows spatial elemental distribution of samples, that is multi-elemental chemical images. Figure 3 shows a chemical map of trace metals, in the ppm range, for a powdered infant formula blended sample [14]. Process integration will be challenging within process environments, however recent developments in LIBS technology and laser confinement techniques offer potential solutions. The approach can also be used for fluids with possible flow through sampling designs feasible. XRF spectrometry is a similar elemental analysis technique but where sample atoms are excited by $\mathrm{X}$-ray photons. Recent development has allowed for portable and process designs which have significant potential in food analysis. As for LIBS integration within process lines will be challenging however XRF process systems are currently operating in other process industries.

\section{Fluid mixing}

Flow mapping within stirred vessels may provide useful insights into the mixing process but may not be suitable for process monitoring or control as many require transparency and/or seeding of particles. Approaches include single-point measuring techniques, which determine the velocity at a set point within the vessel or whole field measuring techniques, which determine the flow pattern simultaneously in a wider region of the bulk of the agitated fluid [15]. Single point measurements include anemometry techniques such as hot wire, laser and phase Doppler. Whole field measurement techniques include particle image velocimetry and planar laser-induced 
Figure 3

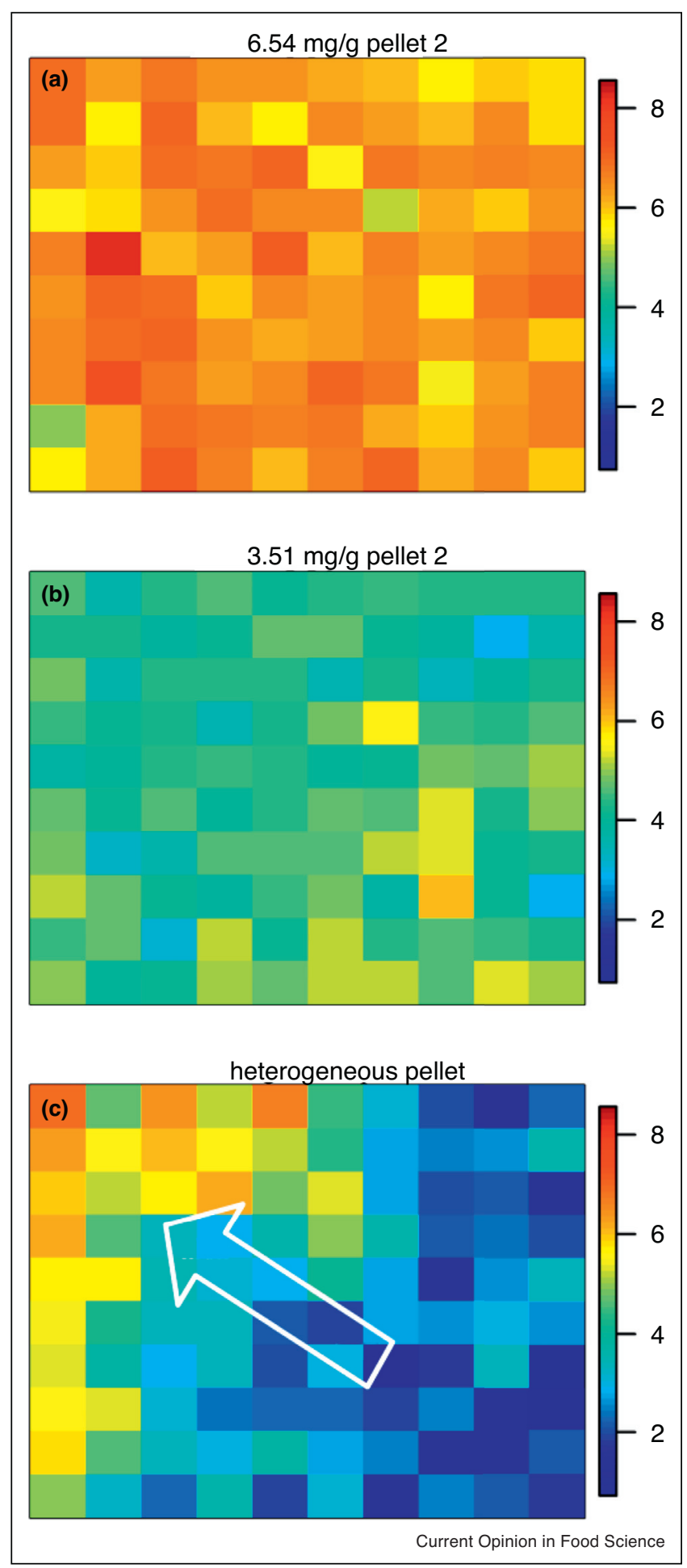

LIBS chemical predicted maps of calcium distribution in powdered infant formula.

fluorescence velocimetry. Current research is actively exploring tomography techniques for mixing insights, monitoring and control.
Figure 4

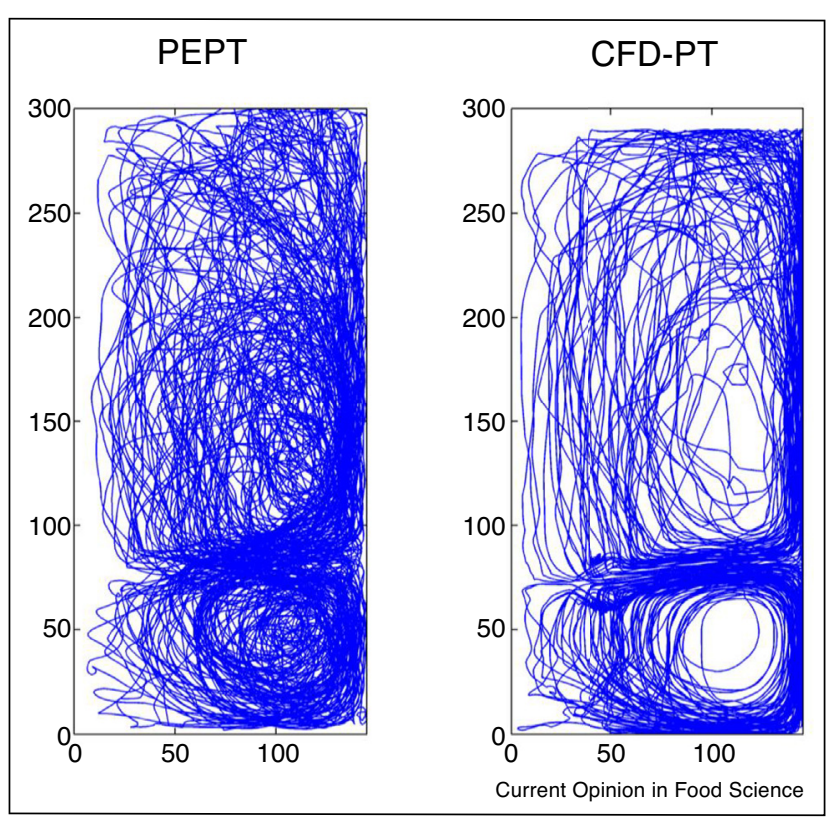

Trajectories obtained after 20 min of PEPT experiments (left hand side) and CFD simulations (right hand side) Trajectories obtained after $20 \mathrm{~min}$ of PEPT experiments (left hand side) and CFD_PT simulations (right hand side). Trajectories projection on a) the rz plane and b) the $r$-th plane.

\section{Tomography techniques}

Tomography is the localised measurement of velocity, density or concentration profiles in $3 \mathrm{D}$, where an image is constructed from an array of sensors surrounding the mixer. Various signals can be used including x-ray, positron and electrical resistance. Positron Emission Particle Tracking (PEPT) employs a single positron-emitting particle as a flow tracer, which is tracked in $3 \mathrm{D}$ space and time to reveal its full Lagrangian trajectory. The technique relies on tracking a single neutrally buoyant particle through the processing system which emits back to back $\gamma$-rays allowing the tracer's location to be triangulated over short time intervals resulting in a 3D velocity profile for the tracer [16]. The method facilitates analysis of both opaque fluids and direct analysis within opaque mixers. The technique is particularly useful for the study of multiphase flows, to map the flow of fluids and particles. Figure 4 shows the typical trajectories obtained from experimental and numerical results. Figure 4 illustrates how the trajectory obtained from the CFD-PT simulation is more regular and uniform than the real trajectory recorded in PEPT. It can also been observed that the tracer in the numerical simulation does not visit the centre of the lower and upper loops. The particles paths obtained from the PEPT data can be combined to create animations that offer clarity on the mechanisms of mixing. Recently the technique was combined with magnetic resonance imaging (MRI) to assess mixing in a SMX static mixer geometry [16]. PEPT was shown to provide 
useful data for analysis of velocity gradients and shear rate distribution within mixers which is particularly useful for understanding the impact of such mixers on sensitive food products. However, the addition of particle tracers or any luminescent material are not appropriate for actual production monitoring or control.

Consequently, electrical tomography techniques are gaining increased attention for potential food mixing applications both for process understanding, system design and process monitoring. Electrical tomography approaches include electrical impedance (EIT), electrical capacitance (ECT) and electrical resistance (ERT), whereby a fluid's electrical property e.g. resistance in the case of electrically-conducting liquids and capacitance for nonconducting fluids are measured using sets of electrodes mounted on the inside of the mixing vessel. Such tomographic techniques can be used to monitor and control mixing processes [17]. The responses from the array of sensors are combined into slices, or 'tomograms' to obtain a 3-D representation of the flow. ERT is capable of noninvasively measuring time-evolving concentration fields, hence mixing rates, within a stirred vessel at high spatial resolution and is particularly useful for non-intrusive quantification of mixing processes of complex suspensions [18]. Many foods display non-Newtonian behaviour including shear thinning and yield stress, such rheological properties often lead to issues including poor mixing, low heat transfer and fouling. ERC is currently providing useful insights into the performance of various impellers and stirred reactors for such complex foods.

Nuclear magnetic resonance (NMR) and magnetic resonance imaging (MRI) are spectroscopic techniques based on the interaction between nuclear magnetic moments and applied external magnetic fields [19]. These techniques are used to measure composition, structure, molecular mobility, molecular diffusion and bulk material motion. MRI is an non-invasive technique suited to obtain both concentration and velocity profiles within process geometries. MRI offers significant potential for mixing as it can be employed for opaque fluids and operates under real-time conditions. The technique has been employed for studies of mixing processes for both batch and continuous systems. Lee et al. [20] employed MRI tomography successfully to quantify the extent of mixing with a fluid and particle model system. In addition to the images, descriptive statistics can be used to distinguish between uniform and non-uniform mixtures. MRI has also been investigated to spatially resolve and quantify mixtures of fine powders.

\section{References}

1. Cullen PJ, Twombly W, Connelly RK, Dickey DS: Mixing in the food industry. In Advances in Industrial Mixing: A Companion to the Handbook of Industrial Mixing. Edited by Kresta SM, Etchells AW, Dickey DS, Atiemo-Obeng VA. Wiley; 2015. ISBN: 978-0-47052382-7.
2. Rielly CD: Mixing theory. In Pharmaceutical Blending and Mixing. Edited by Cullen PJ, Abatzoglou N, Rielly CD. 2015.

3. Jamrógiewicz M: Application of the near-infrared spectroscopy in the pharmaceutical technology. J Pharm Biomed Anal 2012, 66:1.

4. De Beer T, Burggraeve A, Fonteyne M, Saerens L, Remon JP, Vervaet C: Near infrared and Raman spectroscopy for the inprocess monitoring of pharmaceutical production processes. Int J Pharm 2011, 417:32.

5. Swarbrick B: Advances in instrumental technology, industry guidance and data management systems enabling the widespread use of near infrared spectroscopy in the pharmaceutical/biopharmaceutical sector. J Near Infrared Spectrosc 2014, 22:157.

6. Grout B: Application of near infrared conformance trending for material quality, container consistency and minimisation of process risk. J Near Infrared Spectrosc 2014, 22:169.

7. Abatzoglou N: Mixing theory. In Pharmaceutical Blending and Mixing. Edited by Cullen PJ, Romañach RJ, Abatzoglou N, Rielly C. Wiley; 2015. ISBN: 047071055.

8. Togashi D, Alvarez-Jubete L, Rifai H, Cama-Moncunill R, Cruise P, Sullivan C, Cullen PJ: Evaluation of diffuse reflectance near infrared fiber optical sensors in measurements for chemical identification and quantification for binary granule blends. $J$ Near Infrared Spectrosc 2015, 23:133-144.

9. Cama-Moncunill R, Markiewicz-Keszycka M, Dixit Y, CamaMoncunill X, Pietat Casado M, Cullen PJ, Sullivan C: Multipoint NIR spectroscopy for gross composition analysis of powdered infant formula under various motion conditions. Talanta 2016, 154:423-430.

10. Vergote GJ, De Beer TRM, Vervaet C et al.: In-line monitoring of a pharmaceutical blending process using FT-Raman spectroscopy. Eur J Pharm Sci 2004, 21:479-485.

11. Hausman DS, Cambron RT, Sakr AA: Application of Raman spectroscopy for on-line monitoring of low dose blend uniformity. Int J Pharm 2005, 298:80-90.

12. De Beer TRM, Bodson $C$, Dejaegher $B$, Walczak B, Vercruyss $P$, Burggraev A, Lemos A, Delattre L, Vander Heyden Y, Remon JP, Vervaet C, Baeyens WRG: Raman spectroscopy as a process analytical technology (PAT) tool for the in-line monitoring and understanding of a powder blending process. J Pharm Biomed Anal 2008, 48:772-779.

13. Kaiser J, Novotný K, Martin MZ, Hrdlička A, Malina R, Hartl M, ...., Kizek R: Trace elemental analysis by laser-induced breakdown spectroscopy - biological applications. Surf Sci Rep 2012, 67:233-243.

14. Cama-Moncunill X, Markiewicz-Keszycka M, Dixit Y, CamaMoncunill R, Casado-Gavalda MP, Cullen PJ, Sullivan C: Feasibility of laser-induced breakdown spectroscopy (LIBS) as an at-line validation tool for calcium determination in infant formula. Food Control 2017, 78:304-310.

15. Mavros P: Flow visualization in stirred vessels: a review of experimental techniques. Chem Eng Res Des 2001, 79:113-127.

16. Mihailova O, Lim V, McCarthy MJ, McCarthy KL, Bakalis S: Laminar mixing in a SMX static mixer evaluated by positron emission particle tracking (PEPT) and magnetic resonance imaging (MRI). Chem Eng Sci 2015, 137:1014-1023.

17. Mosorov V: Applications of tomography in reaction engineering (mixing process). In Industrial Tomography. Edited by Wang M. 2015:509-528. ISBN: 978-1-78242-118-4.

18. Kazemzadeh A, Ein-Mozaffari F, Lohi A, Pakzad L: Intensification of mixing of shear-thinning fluids possessing yield stress with the coaxial mixers composed of two different central impellers and an anchor. Chem Eng Process: Process Intens 2017, 111:101-114.

19. Lim V, Hobby AM, McCarthy MJ, McCarthy KL: Laminar mixing of miscible fluids in a SMX mixer evaluated by magnetic resonance imaging (MRI). Chem Eng Sci 2015, 137:1024-1033.

20. Lee $\mathrm{Y}, \mathrm{McC}$ arthy MJ, McCarthy KL: Extent of mixing in a twocomponent batch system measured using MRI. J Food Eng 2001, 50:167-174. 